\title{
A repertoire of protease inhibitor families in Amblyomma americanum and other tick species: inter-species comparative analyses
}

\author{
Lindsay M. Porter, Željko M. Radulović and Albert Mulenga*
}

\begin{abstract}
Background: Protease inhibitors (PIs) are important regulators of physiology and represent anti-parasitic druggable and vaccine targets. We conducted bioinformatic analyses of genome and transcriptome data to determine the protease inhibitor (PI) repertoire in Amblyomma americanum and in 25 other ixodid tick species. For A. americanum, we compared the PI repertoires in fed and unfed, male and female A. americanum ticks. We also analyzed PI repertoires of female 48, 96 and 120 h-fed midgut (MG) and salivary gland (SG) tissues.

Results: We found 1,595 putative non-redundant PI sequences across 26 ixodid tick species. Ticks express Pls from at least 18 different families: 11, 12, 14, 18, 121, 125, 129, 131, 132, 135, 139, 143, 151, 153, 163, 168, 172 and I74 (MEROPS). The largest PI families were 12,14 and 18 and lowest in 121, 131, 132, 135 and 168. The majority (75\%) of tick Pls putatively inhibit serine proteases, with $\sim 11$ and 9\% putatively regulating cysteine or metalloprotease-mediated pathways, respectively, and $\sim 4 \%$ putatively regulating multiple/mixed protease types. In A. americanum, we found 370 PIs in female and 354 in male ticks. In A. americanum we found 231 and 442 in unfed and fed ticks, respectively. In females, we found 206 and 164 PIs in SG and MG, respectively. The majority of highly cross-tick species conserved Pls were in families 11, 12, 18, 121, 125, 129, 139 and 143.

Conclusions: Ticks appear to express large and diverse repertoires of PIs that primarily target serine protease-mediated pathways. We speculate that PI families with the highest repertoires may contain functionally redundant members while those with the lowest repertoires are functionally non-redundant Pls. We found some highly conserved Pls in the latter category, which we propose as potential candidates for broad-spectrum anti-tick vaccine candidates or druggable targets in tick control.
\end{abstract}

Keywords: Protease inhibitors in ticks, Amblyomma americanum, Hard ticks

\section{Background}

Ticks are a significant source of morbidity and mortality in both public health and veterinary medicine. Ticks may transmit a wide diversity of pathogenic organisms to their hosts including viruses, bacteria, protozoa and helminthes [1]. In the United States alone, there are at least 15 human diseases for which the associated pathogens are transmitted by ticks [2]. Between 1982 and 2001, the list of human diseases caused by tick-vectored pathogens grew substantially with the addition of 15 new bacterial pathogens [3]. Of veterinary concern, the major diseases caused

\footnotetext{
* Correspondence: amulenga@crm.tamu.edu

Department of Veterinary Pathobiology, Texas A\&M University College of Veterinary Medicine and Biomedical Sciences, 4647 TAMU, College Station, TX 77843, USA
}

by tick-transmitted pathogens are Lyme, babesiosis, theileriosis, heartwater, anaplasmosis, cytauxzoonosis and hepatozoonosis $[4,5]$. In livestock production, an estimated $80 \%$ of the world's cattle populations are affected by disease-causing tick-borne pathogens [6]. Aside from transmitting disease agents, ticks themselves can lead to a variety of indirect veterinary and medical morbidity including toxicosis, paralysis, anemia, wounds susceptible to bacterial or screwworm fly infections, damages to hides and even death [4]. Limitations to acaricide use are the resistance to these chemicals, environmental contamination, cost of development for new chemicals and also food contamination [7-11]. Immunization of hosts against tick feeding is a validated alternative to acaricide-based tick control $[9,12]$. However, the major bottleneck to the 
development of anti-tick vaccines is the discovery of effective target antigens. Protease inhibitors are among some of the most attractive potential anti-tick vaccine antigens.

The balance between proteases and protease inhibitors is essential for the regulation of normal homeostasis across life: from microbes, to plants, to animals. Throughout taxa, essential pathways are regulated by proteases [13-18]. Left uncontrolled, however, protease activity can result in diseases such as cancer, emphysema and blood coagulation disorders [19-23]. Thus, to avoid aberrant protease activity, protease inhibitors tightly and precisely control protease activity. In parasites including ticks, protease inhibitor function is viewed from two perspectives: one being their significance in regulating the homeostasis of the tick itself, and the other being their significance in regulating the parasite-host interaction.

Ticks accomplish feeding by disrupting host tissue and then imbibing blood that bleeds into the wounded area. Tick feeding provokes the host's protease-mediated defense pathways including inflammation, blood clotting and platelet aggregation. Thus, ticks were proposed to secrete protease inhibitors to evade these defense mechanisms, making them prime candidates for anti-tick vaccine studies [24, 25]. Prior to the advent of DNA technology, efforts were focused on purifying proteases and their inhibitors from crude tick protein extracts for medicinal applications [26-28]. However, many tick cDNAs encoding putative protease inhibitors have now been cloned from multiple tick species [24, 29-35]. Additionally, with the recent sequencing of the I. scapularis genome [36], and of several tick transcriptomes [37-49], the focus of tick protease inhibitor research can shift from discovery to characterization. Data mining of sequences from these studies, as available in public databases, has revealed that tick genomes, like many other organisms, encode for high numbers of both proteases and protease inhibitors. Although proteases and their inhibitors are attractive anti-tick vaccine antigens, high numbers of sequences in certain groups of PI families suggests possible functional redundancy. Redundant systems are a potential problem in that targeting one member may result in ticks switching to a functionally equivalent substitute. Therefore, a more detailed bioinformatic investigation into these sequences might reveal a better prioritization plan for vaccine candidate selection. To this end, the first step and the goal of this study was to organize and prioritize the protease inhibitors, into redundant and least or non-redundant systems.

In this study we used Amblyomma americanum inhouse transcriptome data, as well as putative protease inhibitor sequences for ixodid tick species that have been deposited in the MEROPS [50], and/or GenBank databases to compile a reference of all reported putative protease inhibitors in ixodid ticks. We found and analyzed
1,595 non-redundant putative PI sequences across 26 ixodid tick species. In $A$. americanum we found evidence to support previous findings of a time-dependent PI expression in salivary glands $[49,51]$ coined as "sialome switch" [52], however our analyses provide evidence of time-dependent PI expression throughout tick tissues. Additionally, our global analysis differentiated tick PI families that are likely redundant or non-redundant, as well as PIs that are conserved across tick species and that may regulate pathways essential in all ticks. This study serves as the first step in prioritizing tick PIs as anti-tick vaccine antigen candidates.

\section{Methods}

\section{Identification of putative tick protease inhibitors (PI)}

Putative tick PI sequences were identified from two sources. A previously assembled $A$. americanum transcriptome (BioProject accession number PRJNA226980) was annotated by local batch blasting against the NCBI protein database and the Conserved Domain Database (CDD) using the CLC Genomics Workbench software vers 8.0.1 (Qiagen, Hilden, Germany) as previously described [53]. Tick PI sequences were also downloaded from the NCBI GenBank database using keyword searches of the database. For PIs not uniquely characterized by a specific domain, annotations were based on $>95 \%$ identity to annotated sequences in databases. PI sequences were also acquired from the MEROPS version 9.4 database (http://merops.sanger.ac.uk/) [50], and noted according to PI family.

Annotated putative PI sequences from each tick species were sorted according to family and compiled into a single file. All files were then subjected to multiple sequence alignments, against themselves, to identify redundant sequences in each file. An identity value of $95 \%$ or greater was deemed as evidence of redundancy, and only one sequence was retained for further analysis. In this way, we determined a non-redundant count for each PI family for each tick species. Sequences where family membership could not be verified on the basis of known domains or similarity to characterized members of the protease inhibitor family were also eliminated from the study.

\section{Identification of putative expression patterns and homologs in other ticks}

To determine apparent expression patterns, putative $A$. americanum tick PI sequences were further screened for presence or absence in different transcriptomes: male and female, unfed and fed whole $A$. americanum ticks, as well as dissected salivary gland (SG) and midgut (MG) of 48, 96 and $120 \mathrm{~h}$ fed female ticks. Identification of highly conserved tick serine protease inhibitors (serpins) in family I4 was previously accomplished [53]. In this study, we determined PIs homologs for all other PI families by BLASTX 
screening of $A$. americanum PIs against other tick PIs of the same family. PI sequences in other tick species where identity values were $\geq 50 \%$ were included in further analyses.

\section{Results and discussion}

Tick genomes likely encode hundreds of protease inhibitors

Additional file 1: Table S1 (please note the different tabs) lists a total of 1,595 putative non-redundant PI sequences across 26 ixodid tick species. Our analysis of our A. americanum transcriptome data revealed 515 putative PIs for this species (Table 1). We also found more than 100 PI sequences for three additional tick species: Ixodes scapularis (239), Ixodes ricinus (149), Amblyomma maculatum (135) and Amblyomma cajennense (106) (Fig. 1). Between ten and 100 sequences were found for eight tick species: Amblyomma triste (83), Rhipicephalus pulchellus (76), Rhipicephalus microplus (57), Rhipicephalus appendiculatus (35), Amblyomma parvum (31), Haemaphysalis longicornis (20), Amblyomma variegatum (20) and Rhipicephalus sanguineus (13) (Table 1). The remaining 13 tick species currently have less than ten reported non-redundant PI sequences: Dermacentor variabilis (8),

Table 1 Amblyomma americanum protease inhibitor counts

\begin{tabular}{|c|c|c|c|c|c|c|c|c|c|c|}
\hline \multirow{2}{*}{$\begin{array}{l}\text { Inhibitor } \\
\text { family }\end{array}$} & \multicolumn{3}{|c|}{ Sex related ${ }^{a}$} & \multicolumn{3}{|c|}{ Feeding related $^{\mathrm{b}}$} & \multicolumn{3}{|c|}{ Tissue distribution $^{c}$} & \multirow[t]{2}{*}{ Total } \\
\hline & $\mathrm{F}$ & M & $F \& M$ & $\mathrm{FD}$ & UF & FD\&UF & SG & MG & SG\&MG & \\
\hline 11 & 2 & 8 & 11 & 8 & 1 & 12 & 3 & 2 & 6 & 21 \\
\hline 12 & 69 & 35 & 73 & 102 & 30 & 45 & 60 & 14 & 27 & 177 \\
\hline $14^{\mathrm{d}}$ & 33 & 57 & 31 & 87 & 12 & 21 & 12 & 10 & 26 & 122 \\
\hline 18 & 6 & 2 & 9 & 6 & 2 & 9 & 5 & 0 & 5 & 17 \\
\hline 121 & 0 & 0 & 1 & 0 & 0 & 1 & 0 & 0 & 1 & 1 \\
\hline 125 & 6 & 10 & 12 & 17 & 0 & 11 & 3 & 3 & 9 & 28 \\
\hline 129 & 7 & 7 & 1 & 12 & 1 & 2 & 1 & 4 & 1 & 15 \\
\hline 131 & 2 & 0 & 4 & 3 & 1 & 2 & 0 & 1 & 4 & 6 \\
\hline 132 & 0 & 0 & 2 & 0 & 0 & 2 & 0 & 0 & 2 & 2 \\
\hline 135 & 1 & 0 & 0 & 0 & 0 & 1 & 0 & 0 & 1 & 1 \\
\hline 139 & 13 & 2 & 13 & 14 & 4 & 10 & 8 & 5 & 9 & 28 \\
\hline 143 & 17 & 10 & 7 & 14 & 11 & 9 & 8 & 7 & 3 & 34 \\
\hline 151 & 0 & 9 & 5 & 9 & 0 & 5 & 1 & 0 & 4 & 14 \\
\hline 163 & 8 & 8 & 32 & 9 & 9 & 30 & 0 & 12 & 7 & 48 \\
\hline $168^{e}$ & 1 & - & - & - & - & - & 1 & - & - & 1 \\
\hline Total & 164 & 148 & 202 & 282 & 71 & 160 & 101 & 59 & 105 & 515 \\
\hline
\end{tabular}

${ }^{a}$ Count of protease inhibitors found present in female (F) or male (M) ticks, and number found present in both females and males (F\&M)

${ }^{b}$ Count of protease inhibitors found present in fed (FD) or unfed (UF) ticks, and number found present in both fed and unfed (FD\&UF)

${ }^{c}$ Count of protease inhibitors found present in salivary glands (SG) or midgut (MG), and number found present in both of these tissues (SG\&MG)

${ }^{\mathrm{d} D a t a}$ reported here are from our previously published analysis of family 14 [53]

Data reported here are from [52] which analyzed female salivary gland data only
Dermacentor andersoni (8), Rhipicephalus haemaphysaloides (7), Ixodes pacificus (7), Ixodes persulcatus (2), Haemaphysalis bispinosa (2), Hyalomma anatolicum anatolicum (2), and one each for Amblyomma hebraeum, Hyalomma marginatum rufipes, Dermacentor silvarum, Ixodes ovatus, Rhipicephalus bursa and Rhipicephalus annulatus. Since five well-studied tick species have more than 100 reported PI sequences, we predict that this will also be the case for other ixodid tick species, once further sequence data becomes available. Interestingly, we found that the large PI counts for A. americanum, A. maculatum, A. cajennense, I. scapularis and $I$. ricinus are consistent with current counts in other wellstudied arthropods, such as Anopheles gambiae (131) Apis mellifera (224), Bombyx mori (269), Culex quinquefasciatus (99), Drosophila melanogaster (166) and Tribolium castaneum (184) [50]. We would like to advise the reader that data presented in this study need further validation using quantitative (q) RT-PCR. Despite this limitation, these data provide important information on protease inhibitors expressed in tick genomes.

\section{Amblyomma americanum PI repertories may vary by sex, tissue and feeding time point}

Evidence in other in silico transcriptome analyses show evidence of sex-based differences in transcript levels during feeding in $R$. appendiculatus [51], and temporal and sex-based differences in transcript repertoires across feeding time points in $R$. pulchellus [49]. Additionally, studies using qRT-PCR analysis have validated spatiotemporal variance of PI expression in ticks [54-56] as well as semi-quantitative RT-PCR analyses showing spatiotemporal differences in PI expression patterns [57-59]. Although our study is entirely in silico, our data support the hypothesis that at least some tick PIs are differentially expressed in tissues, time points and possibly between the sexes. In our transcriptome data we found that of $515 \mathrm{~A}$. americanum PI sequences, 164 ( 32\%) and 148 ( 29\%) transcripts were found exclusively in females and males, respectively, while $202(\sim 39 \%)$ were found in both sexes (Table 1, Fig. 2). The next step will be to further explore the hypothesis that $A$. americanum ticks express sex-specific PIs using qRT-PCR.

In $A$. americanum SG and MG tissues at 48, 96 and $120 \mathrm{~h}$ of feeding, we found a total of 265 PIs across 14 families (Table 1, Figs. 2, 3). It is interesting to note that we found more PI transcripts in SG (205) than in MG (162). Also interesting is that of the 515 total A. americanum PIs, we could not find 250 in either SG or MG tissues. While technical errors could explain the lack of PIs in these tissues, the data suggests the possibility that at least some PIs are not expressed in these tissues, and that they might be expressed in other tissues that are not primarily exposed to the host components. Although data here need further validation using qRT-PCR, the 250 sequences not found 


\section{A. americanum}

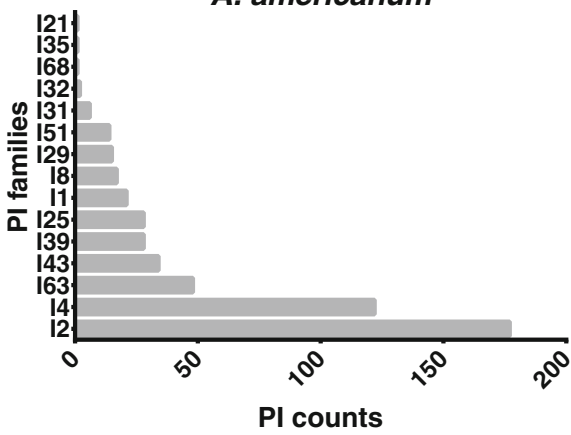

I. scapularis

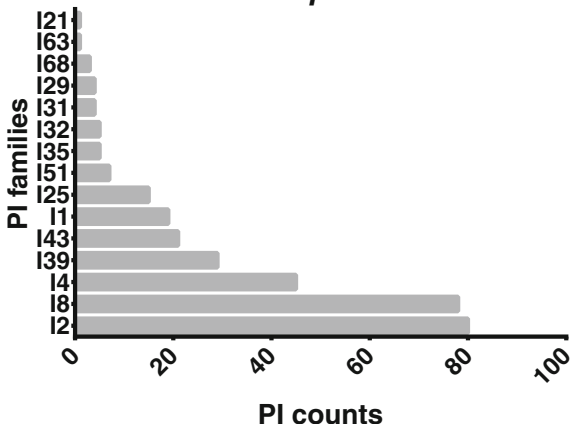

A. maculatum

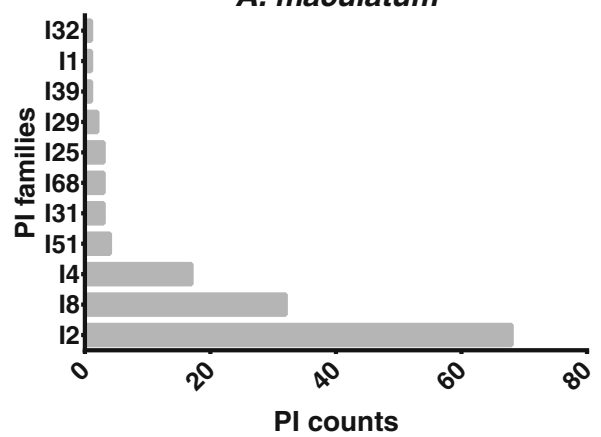

I. ricinus

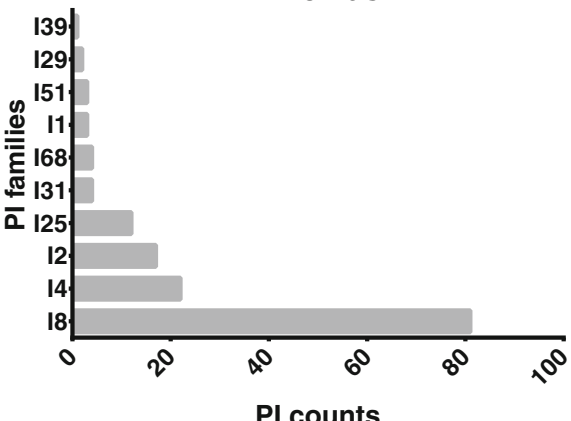

Fig. 1 PI repertoire for four major medically important tick species. The count of protease inhibitors (PIs) in each PI family for Amblyomma americanum, Amblyomma maculatum, Ixodes scapularis and Ixodes ricinus are shown. PI families where total count was less than ten were excluded from the graphs

in both SG and MG may not be priority candidates for anti-tick vaccine development. Data for individual families are outlined in the following sections. We would like to note that data here are qualitative and not quantitative; therefore, it is possible that distribution patterns for $A$. americanum PIs may change after further analyses.

\section{Majority of tick PIs putatively inhibit serine protease- mediated pathways}

Figure 3 shows that the majority $(\sim 72.5 \%, 1,159 / 1,595)$ of tick PI sequences in this study belong to families inhibiting serine proteases and are therefore putative inhibitors of serine proteases: 527 in I2 (Kunitz-like serine protease inhibitors), 287 in I4 (serine protease inhibitors, [serpin]), 237 in I8 (TIL domain elastase inhibitors), 61 in I1 (Kazal), 31 in I51 (PEBP, phosphatidylethanolaminebinding protein), 10 in I53 (madanin), three in family I21 (secretogranin), two in I72 (chimadanin) and one in I74 (variegin, thrombin inhibitor) (Additional file 2: Table S2 and Fig. 1). Of the remaining 436 PI sequences, 164 ( 13\%) belong to families inhibiting cysteine proteases: 102 in I25 (cystatin), 53 in I31 (thyropin), 39 in I29 (CTLA, Cytotoxic T-lymphocyte antigens) and 12 in $\mathrm{I} 32$ (survivin); and 134 ( 10.5\%) belong to families of metalloprotease inhibitors: 75 in I43 (oprins), 62 in I63 (pro-eosinophil major basic protein), 24 in I68 (tick carboxypeptidase inhibitor) and
6 in I35 (tissue inhibitor of metalloproteinases). The remaining 63 PIs $(\sim 4 \%)$ are in family I39, alpha-2macroglobulins, which are non-specific PIs.

The relative PI family distribution we observed at the global level was mirrored at the individual tick species level with most PIs belonging to families inhibiting serine proteases. We would like to note that some tick serpins may inhibit both cysteine and serine proteases, which would change the relative proportions of proteases inhibited ([60]; unpublished observations by the authors). Nevertheless, such a high number of putative inhibitors of serine proteases suggest that inhibitors of serine proteases play an important and extensive role in regulating tick physiology.

In combination with our observations that ticks express high numbers of PIs for some families, these data suggest a potential for highly functionally redundant systems, whereby several proteins can accomplish the same physiological goal. Evidence of functional redundancy could signal that those PIs regulate tick physiological pathways that must be functional without fail, and thus finding ways to block such PIs could prove to be highly effective in tick control. On the other hand, PI families with fewer sequences could be interpreted as less likely to have extensive functional redundancy and these proteins could be the most attractive targets for anti-tick vaccine development. 


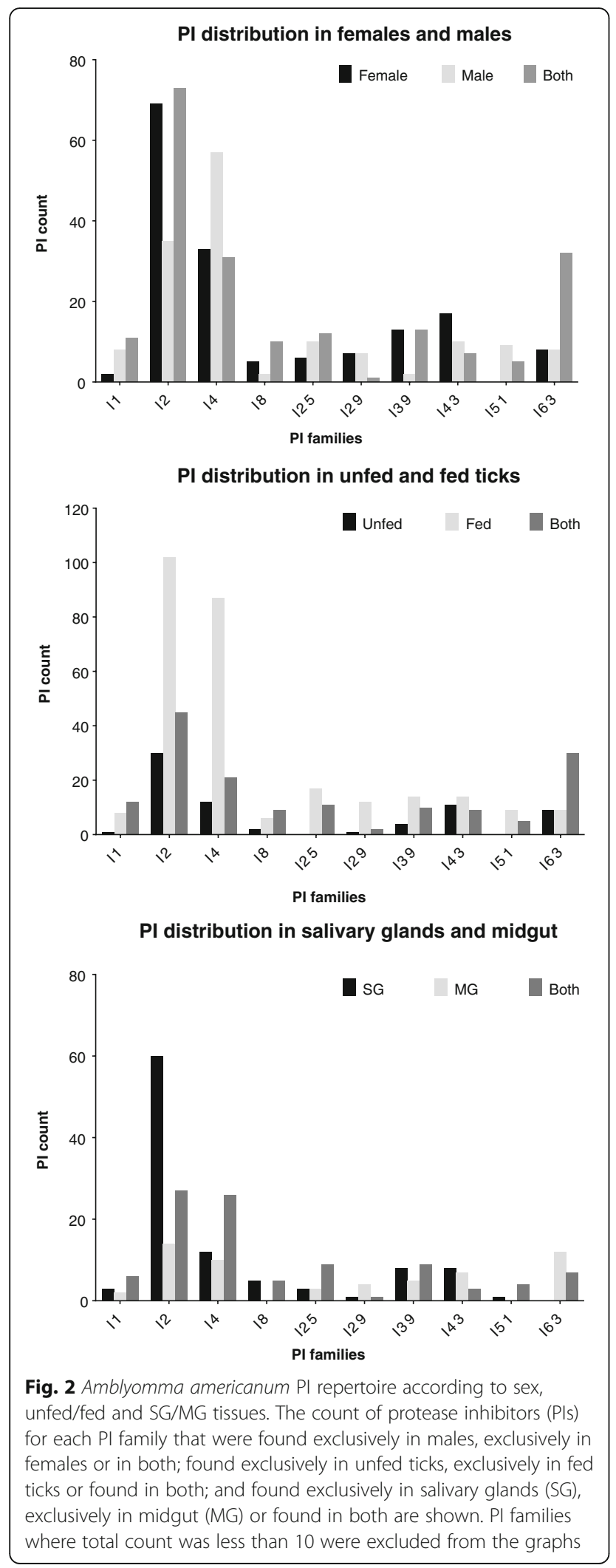

Families of Pls encoded in A. americanum and other tick species

\section{Family 11: Kazal}

In $A$. americanum we found 21 non-redundant Kazal protease inhibitor (KPI) sequences (Table 1). In our transcriptome analysis we found the majority of these $(19 / 21)$ in male ticks, while we detected a little more than half $(13 / 21)$ in female ticks (Table 1). It is interesting to note that eight KPI transcripts were found only in males, therefore future experiments should aim to validate whether or not these PIs are differentially expressed in the sexes. Strikingly, in our data we found almost all KPIs $(20 / 21)$ in fed ticks, strongly supporting the hypothesis that many KPIs function in feeding physiology.

In 11 other ixodid tick species we found 33 putatively non-redundant KPI sequences (Additional file 1: Table S1): 24 for I. scapularis, four for I. ricinus, three for A. variegatum, two for $A$. cajennense and only one KPI sequence for each of $A$. maculatum, A. triste, A. parvum, $R$. microplus, $R$. sanguineus, $R$. pulchellus and $H$. longicornis. Our interspecific BLAST analyses showed 13 A. americanum KPIs to be highly conserved in other ixodid tick species, primarily $I$. scapularis (Additional file 2: Table S2). Amino acid identities exceeded 65\% for six A. americanum Kazal PIs with four of these ranging between 81 and $98 \%$. Interestingly, we found these four very highly conserved PIs to be expressed either in all or almost all tissues at all of the time points examined in this study (Additional file 2: Table S2). Six of the seven less-conserved KPIs (identities of 50-66\%) had a more limited tissue/time point/sex distribution. Notably, three of these six were found only in fed males.

The only functional data currently available for tick KPIs is from H. longicornis (ABB76182.1) and is a follistatinrelated protein (FRP, homologous to human FRP), binding

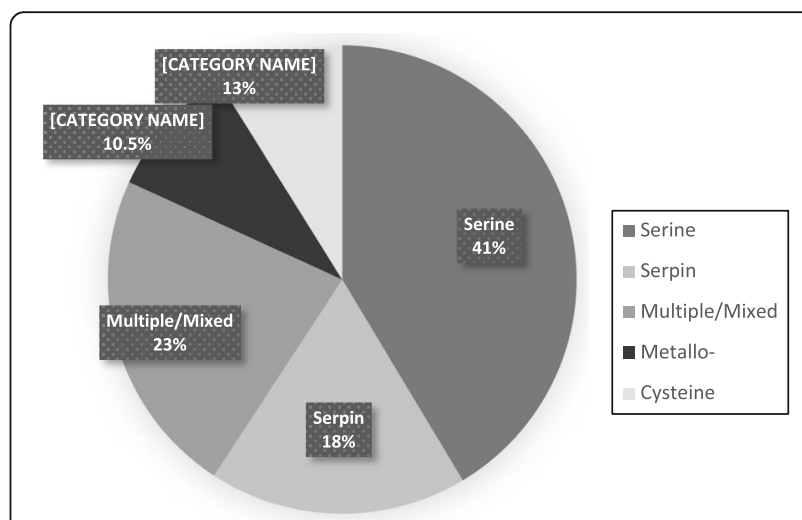

Fig. 3 Proportions of tick protease inhibitors (PIs) which are predicted inhibit each protease catalytic type. Tick PIs with putative activity against serine or multiple/mixed activity represent the vast majority of tick PIs. Serpins are shown separately from other PI families showing activity against multiple catalytic types to illustrate that most tick PIs likely do inhibit proteases of the serine catalytic type 
activin A and BMP-2 [61]. Activin has been implicated in cell growth and differentiation, wound repair and acute inflammation in the innate immune response $[62,63]$. We found one ABB76182.1 homolog in A. americanum with $84 \%$ identity. Several inhibitory KPIs from other hematophagous organisms have been described. Bdellins from the medicinal leech Hirudo medicinalis are Kazal-type inhibitors of trypsin and plasmin [64]. Additionally, three Kazal-type thrombin inhibitors from hematophagous triatoma bugs have been described: dipetalogastin [65], infestin [66] and rhodniin, [67]. In ticks, several proteins containing Kazal domains have been reported including an organic anion transporting polypeptide (Oatp), from A. americanum [68] and from I. scapularis [69], as well as insulin growth factor binding proteins from $R$. appendiculatus, $R$. microplus and A. variegatum [47]. Inhibitory properties for tick KPIs however have not been reported. Given the important role KPIs play in facilitating hematophagy in other organisms, it will be interesting to investigate if tick KPIs function the same.

\section{Family 12: Kunitz}

In $A$. americanum we found 177 non-redundant Kunitz sequences. In our transcriptome data we found many Kunitz sequences only in female ticks (69) and many only in male ticks (35), while 73 were confirmed to be expressed in both males and females, again suggesting there may be differential expression of this PI family between the sexes. Also noteworthy is the 60 Kunitz PIs found only in SG suggesting there may be at least some Kunitz PIs in A. americanum that are only expressed in SG. The 87 Kunitz sequences found in SG shows the complexity of tick salivary gland physiology. Notably, similar numbers of Kunitz sequences were found only in unfed females (30) as were found common to both fed and unfed females (45), while more than double those numbers were found only in fed female ticks (102). These preliminary data support a hypothesis that at least some Kunitz sequences may be expressed only in fed ticks and this hypothesis should be confirmed with qRT-PCR analysis. Such a marked diversity of Kunitz sequences during feeding demonstrates their importance in tick feeding physiology. In 17 other ixodid tick species we found 350 putatively nonredundant Kunitz sequences (Additional file 1: Table S1): 117 for I. scapularis, 72 for A. maculatum, 36 for A. triste, 32 for $A$. cajennense, 22 for $R$. pulchellus, 17 for I. ricinus, nine for $R$. microplus, seven each for I. pacificus, $A$. variegatum and $A$. parvum, six each for $H$. longicornis and $R$. sanguineus, four for $D$. andersoni, three for $D$. variabilis, two each for $R$. appendiculatus and $R$. haemaphysaloides, and one for A. hebraeum. Our BLASTX analyses revealed $12 \mathrm{~A}$. americanum Kunitz PIs homologs in other tick species with identities at $\geq 60 \%$ (Additional file 2: Table S2). Interestingly, all except one of these 12 were found in fed ticks, supporting the hypothesis that conserved $A$. americanum PIs play an important role during feeding. However, it is worth mentioning that four of 12 were confirmed by our transcriptome data to be expressed in all or almost all tissues and time points examined in this study.

Kunitz is a large family found in ticks and other hematophagous species [50, 70-73]. Accordingly, we found that 18 of 26 tick species in this study had Kunitz sequences deposited in GenBank (Additional file 1: Table S1). Kunitz proteins are serine protease inhibitors of the S1 protease family [50]. Two Kunitz inhibitors from I. scapularis have been functionally characterized: Ixolaris (AAK83022) [74] and penthalaris (AAM93636) [75]. Ixolaris and penthalaris are tissue factor pathway inhibitors, inhibiting blood clotting via FVIIa/TF binding [74, 75]. Many Kunitz-type inhibitors from other tick species have also been described, and are now known to inhibit a range of proteases including thrombin, trypsin, plasmin, kallikrein, neutrophil elastase and FXa [70]. Some well-described examples of tick Kunitz inhibitors include the thrombin-induced platelet aggregation inhibitors savignygrin (Ornithodoros savignyi, AAM54048), [76], disagregin (O. moubata, AAB30092), [77, 78] and the thrombin inhibitors savignin (O. savignyi, AAL37210), [79], boophilin ( $R$. microplus, CAC82583), [80], amblin (Amblyomma hebraeum, AAR97367), [81] and hemalin (H. longicornis, BAH02683), [82]. Additionally, a trypsininhibiting Kunitz protein from $D$. andersoni shows bacteriostatic properties and is upregulated during infection with Rickettsia [83] demonstrating a role for Kunitz inhibitors aside from facilitating hematophagy. The widespread nature of this family across tick species and its apparent role in feeding and defense indicate the likelihood that all ticks express Kunitz inhibitors. However, the large number of Kunitz inhibitors suggests that this family is likely to contain functionally redundant members.

\subsection{Family 14: Serpins}

A previous thorough analysis of serine protease inhibitors (serpins) in A. americanum shows 122 unique serpin sequences for this species [53]. In ten other tick species we found 165 putatively non-redundant serpin sequences: I. scapularis (45), $R$. appendiculatus (29), $R$. microplus (27), I. ricinus (22), A. maculatum (17), R. pulchellus (16), A. variegatum (4), R. haemaphysaloides (2), I. persulcatus (2) and H. longicornis (1) (Additional file 1: Table S1). Serpins were originally identified as inhibitors of serine proteases [84]. While other PIs inhibit serine proteases, this family is differentiated from other PIs by the RCL of the serpin domain. Further functional studies show certain serpins have cross-class inhibitory functions against cysteine proteases [85-87]. For additional reading, we refer the reader to Porter et al. [53]. 


\section{Family 18: Chymotrypsin/Elastase Inhibitor}

In $A$. americanum we found 17 non-redundant I8 inhibitor sequences. Our transcriptome data confirmed the expression of more than half of these (nine of 17) in both male and female ticks, while six were found only in females and two were found only in males. Our data also confirmed the expression of more than half (nine of 17) of I8 PIs in both fed and unfed ticks, while six were found only in fed ticks and two only in unfed ticks. We also found 10 sequences in SG, five of which were only in SG while the other five were also in MG. Based on these data it will be interesting to explore the hypothesis of differential I8 PI expression using qRT-PCR data (Fig. 2, Table 1).

In 10 other ixodid tick species we found 220 nonredundant family I8 sequences (Additional file 1: Table S1): 81 in I. ricinus, 78 in I. scapularis, 43 in A. cajennense, 35 in A. triste, 32 in A. maculatum, 21 in A. parvum, 5 in $R$. microplus, 1 in $H$. marginatum rufipes, 1 in $R$. pulchellus and 1 in A. variegatum (Additional file 1: Table S1). Based on the currently available data, family I8 appears to make up a much larger proportion of the Ixodes PI repertoire than the Amblyomma repertoire (Fig. 1). In A. americanum we found seven 18 sequences conserved in other tick species, with amino acid identities ranging from 50 to $72 \%$ (Additional file 2: Table S2). Of note is the $A$. americanum $\mathrm{I} 8$ sequence with $72 \%$ identity to $R$. microplus ixodidin, which is an antimicrobial peptide found in tick hemocytes [88].

There are currently few functional studies for family I8 members in ticks, however in $R$. microplus, inhibitory properties have been verified for some I8 members. These include ixodidin, a single TIL domain chymotrypsin/elastase inhibitor with antimicrobial properties [88], and BmSI-7, another single TIL domain protein shown to inhibit the bacterial protease subtisilin A and the fungal protease Pr1 [89]. Also, a recent study reported a TIL domain protein from $I$. scapularis nymphs as being upregulated and secreted at $48 \mathrm{~h}$ post-attachment [90]. Additionally, an I8 family member from I. ricinus, also upregulated after feeding, shows similarity to von Willebrand Factor [91] and thus may play a role in platelet aggregation inhibition [92]. In the A. maculatum sialotranscriptome 85 CDS (57 complete) that contain TIL domains (or without a TIL domain but having similarity to such sequences) were reported [45] however, redundancy analysis data were not provided.

\section{Family 121: Secretogranin}

In $A$. americanum we found only one non-redundant I21 sequence. Strikingly, our transcriptome data confirmed the expression of this sequence in every tissue and time point, with the exception of SG at 48 and $120 \mathrm{~h}$. Since this sequence is expressed in males, females, unfed ticks and fed ticks in multiple tissues, we speculate that this protein may serve an important role. We also found only one non-redundant I21 sequence in I. scapularis and $D$. variabilis (Additional file 1: Table $\mathrm{S} 1$ ). Interestingly, the $A$. americanum sequence shows $92 \%$ identity to the $D$. variabilis sequence (ACJ12615.1), and 69\% identity to the I. scapularis sequence (Additional file 2: Table S2). The I. scapularis and D. variabilis sequences share $75 \%$ identity.

Family I21 members are inhibitors of serine endopeptidases [50] and have a secretogranin_V domain (GenBank). The type sequence for this family is a neuroendocrine protein from humans, named 7B2, which inhibits the prohormone convertase PC2 [93]. Accordingly, the I21 sequence in $D$. variabilis was found in the synganglion (NCBI), and bioinformatic annotations of these sequences are as neuroendocrine proteins. However, functional studies of tick secretogranins have not yet been reported. The apparent tissue and time point ubiquity and lack of family expansion for $A$. americanum secretogranins makes this family very intriguing, particularly since the pattern of a single secretogranin sequence seems to extend to other tick species. Single- or low-member PI families are interesting from the perspective of their being targeted for control strategies, since it indicates a potential lack of functional redundancy for these proteins.

\section{Family 125: Cystatins}

In $A$. americanum we found 28 non-redundant cystatin sequences. Our transcriptome data confirmed the expression of 15 cystatins in SG and MG (Table 1). We did not find transcripts for all 28 cystatins in all tissues and time points or in both sexes. We found only 18 in females and only 22 in males. It will be interesting to use qRT-PCR data to explore the possibility of differential expression of cystatins in A. americanum. It is interesting to note however, that all cystatins we found to be expressed in unfed male and in unfed female ticks were also found in fed ticks. These data support the hypothesis that ticks likely do not express any cystatins only in the unfed stage.

In 12 other ixodid tick species we found 74 cystatins: I. scapularis (15), I. ricinus (14), A. cajennense (12), $R$. pulchellus (9), $R$. microplus (7), H. longicornis (5), A. maculatum (3), R. sanguineus (3), D. variabilis (2), and one each for A. variegatum, $R$. haemaphysaloides, I. ovatus and $D$. silvarium (Additional file 1: Table S1). Inter-species BLASTX analyses showed eight $A$. americanum cystatins have homologs across multiple tick species with amino acid identities ranging from 50 to $82 \%$ (Additional file 2: Table S2). This makes them interesting targets for antitick vaccines that would be capable of protecting against many species of ticks.

Cystatins are papain-like cysteine protease inhibitors [50]. Functional data show that silencing of A. americanum cystatin RNA results in reduced tick engorgement weights and failure to feed [94]. In $H$. longicornis, the cystatin Hlcyst-2 is expressed highest in MG and hemocytes, shows 
increased expression at feeding, and has been implicated in tick immunity due to inhibitory effects on the growth of Babesia bovis [95]. In I. scapularis, the cystatin sialostatin-1 is a cathepsin $\mathrm{L}$ inhibitor involved in anti-inflammation and inhibition of cytotoxic T-lymphocyte proliferation [96].

\section{Family 129: Cytotoxic T-Lymphocyte Antigen (CTLA)}

Family I29 is also known as cytotoxic T-lymphocyte antigen-2 alpha, (CTLA-2). In A. americanum we found 15 non-redundant CTLA sequences (Table 1). Interestingly, eight CTLAs in males were not found in females and eight in females were not found in males. Additionally, 14 of 15 CTLAs were found in fed ticks, with only two of those 14 found in unfed ticks. Similarly, we found only six of 15 CTLA sequences in SG and MG tissues. Taken together, these data suggest the potential of differential PI expression across tissues and time points that should be further investigated. Most striking in our transcriptome data was that unlike most other PI families, we found almost all CTLA sequences in only a single tissue and time point. While this could be explained by a technical rather than biological reason, it is nonetheless worth noting and worth following up with qRT-PCR expression analysis.

In 12 other ixodid tick species we found 24 putatively non-redundant CTLA sequences: four each for I. scapularis and $R$. appendiculatus, two each for I. ricinus, $R$. haemaphysaloides, A. maculatum, A. variegatum, D. variabilis and $H$. anatolicum anatolicum, and one each for $R$. microplus, $R$. annulatus, $R$. pulchellus and H. longicornis (Additional file 1: Table S1). Inter-specific BLAST analyses revealed eight $A$. americanum CTLAs with homologs in other tick species (Additional file 2: Table S2). CTLAs are unique cysteine proteases inhibitors, where inhibition of the peptidase is via the peptidase propeptide [50]. Therefore, these proteins have a domain profile that includes the I29 inhibitor domain, followed by a $\mathrm{C} 1 \mathrm{~A}$ peptidase domain. Strikingly, we found a high number of tick CTLAs with $60 \%$ or more identity to A. americanum CTLAs, and in every case, the region conserved between sequences included both the I29 inhibitor domain and the protease domain regions. The MEROPS database reports that the I. scapularis genome lacks family I29 homologs, however, we found four non-redundant I. scapularis sequences showing I29 inhibitor domains in the NCBI database (Table 1). While two of the most conserved $A$. americanum CTLAs were not confirmed in our data to be expressed in MG tissues, their homologs in other ticks (AAO60045, -46, -48, ACF35530 and XP_002403652) are characterized as midgut cysteine proteinases. Further investigation is required to determine whether or not $A$. americanum expresses these CTLAs in $M G$.

In mice, Drosophila and Bombyx mori CTLAs are inhibitors of cathepsin-L cysteine proteases [97-99]. Our BLAST analyses revealed that one $A$. americanum CTLA and 12 CTLAs from 10 other tick species show 40-62\% identity (Additional file 2: Table S2) to the I29 inhibitor and cathepsin L protease regions of a CTLA in Sarcophaga peregrina (flesh fly, BAA76272) involved in clearing foreign proteins in this insect [100]. The role of CTLAs in tick physiology remains unknown, however it will be interesting to investigate the potential for tick CTLAs to be involved in eliminating the excess proteins in blood meals or unwanted proteins expressed by tick-borne disease agents.

\section{Family 131: Thyropins}

Family I31 is known as equistatin inhibitory unit 1 and members are called thyropins due to their thyropin (TY) domains [50, 101]. Thyropins are inhibitors of papain-like cysteine proteases and cathepsin-D [50, 101, 102]. In $A$. americanum we found six non-redundant thyropin sequences (Table 1). Unlike most of the other 13 families we analyzed in this study, our data showed that transcripts expressed in males were also expressed in females. Similarly, our data showed that transcripts expressed in SG were also expressed in MG, with the exception of one transcript that was found in neither tissue. Since our data do not support an hypothesis that thyropins play a role in SGspecific physiology, these PIs may not be recommendable as anti-tick vaccine candidates.

In nine other ixodid tick species we found 47 putatively non-redundant thyropins: 16 for $A$. cajennense, seven for $A$. triste, six for $I$. ricinus, five for $A$. maculatum, four each for $I$. scapularis and $R$. pulchellus, three for $R$. microplus, and one each for $A$. variegatum and $R$. sanguineus in public databases (Additional file 1: Table S1). Our BLASTX searches show four of six $A$. americanum thyropin have homologs in other tick species (Additional file 2: Table S2). One interesting observation is that the two most highly conserved $A$. americanum thyropins, homologs of an $A$. variegatum thyropin (DAA34697) at 73 and $85 \%$ identity values, were found only in fed males and in SG and MG of female ticks. It will be interesting to further investigate with qRT-PCR if these thyropins are only expressed in SG and MG tissues in males and females.

Thyropin domains are found in many types of proteins including in saxiphilin, which binds the neurotoxin saxitoxin [103], in testican, which is a cathepsin-L inhibitor and regulates some matrix metalloproteases (MMPs) [104] and in nidogen which functions in basement membrane formation [105] and neutrophil chemotaxis [106]. The functional role of thyropins in ticks is not yet known.

\section{Family I32: Inhibitor apoptosis (IAP)}

In $A$. americanum, we found two non-redundant IAP sequences (Table 1). Interestingly, our transcriptome data confirm expression of both IAPs in fed and unfed male and female ticks, and in both SG and MG tissues. The 
wide distribution of these IAPs implies their importance in $A$. americanum physiology. In five other ixodid tick species we found 10 non-redundant IAPs: five for I. scapularis; two for A. triste; and one each for A. maculatum, $R$. microplus and $R$. pulchellus (Additional file 1: Table S1).

IAPs are also referred to as BIRC or BIR (baculovirus inhibitor repeat containing) proteins. Since there are many sequence annotations in GenBank that include the terms "apoptosis inhibitor," but do not have BIR domains and are not protease inhibitors, we counted only sequences showing a BIR domain in CDD searches. Our two $A$. americanum IAPs each had a homolog in another tick species (Additional file 2: Table S2). Notably, one sequence showed 70\% identity to $R$. microplus AIT40207 while the other sequence had $54 \%$ identity to $I$. scapularis survivin (XP_002413809.1). IAPs inhibit caspases and cysteine endopeptidases [50, 107]. According to CDD search analyses in this study, some tick IAPs also have a C-terminus really interesting new gene (RING) domain also found in other IAP proteins [108, 109]. It has been shown that RING domains in IAPs allow them to ubiquinate caspases for degradation and removal [102]. Based on our analysis, tick IAPs with RING domains are found in I. scapularis, A. triste, R. microplus and $R$. pulchellus. However, the function of IAPs in tick biology remains to be investigated.

\section{Family 135: Tissue inhibitor of metalloproteinases (TIMPs)}

Family I35 is referred to as TIMP (tissue inhibitor of metalloproteinases) [50]. In A. americanum we found only one TIMP sequence (Table 1). In our transcriptome data we found just four redundant TIMP contigs, all of which were found only in females in our transcriptome data. The $A$. americanum TIMP was found in both SG and MG, and in both fed and unfed females. It will be interesting to confirm the lack of TIMP expression in male ticks using qRT-PCR data. We found only five TIMPs for other ixodid tick species, all in I. scapularis (Additional file 1: Table S1). BLASTX analyses show one of these three sequences to have $68 \%$ to the $A$. americanum TIMP (Additional file 2: Table S2). These data suggest ticks have a small TIMP inhibitor repertoire that may not be present at all in some species.

TIMPs are metalloendopeptidase inhibitors [50, 110]. In humans TIMPs are inhibitors of matrixins, which function in tissue remodeling, inflammation and cancer pathogenesis [111, 112]. Additionally, TIMPs are apoptosis and angiogenesis regulators [113]. From the perspective of tick feeding, which occurs over a period of several days, blocking tissue remodeling and repair is essential to maintain the feeding site. Therefore, tick TIMPs as woundrepair inhibitors is an interesting avenue for future research. Furthermore, since our analyses show ticks appear to have few TIMP sequences, these PIs might be involved in lowredundancy or no-redundancy pathways. However, the role of TIMPs in the biology of ticks or of any hematophagous organism remains to be explored.

\section{Family I39: Alpha2-macroglobulins, (a-2 M)}

In $A$. americanum we found 28 non-redundant alpha2macroglobulins $(\alpha-2 \mathrm{M})$ sequences (Table 1$)$. Our transcriptome data confirmed the expression of the majority of these $28(26 / 28)$ in female ticks, while we found only a little more than half $(15 / 28)$ in male ticks. Similarly, in SG and MG tissues we found 18 of $28 \alpha$-2Ms, and confirmed expression of nine in both tissues. As our data support that these inhibitors are expressed in many different tick tissues, this is an indication that they may serve diverse functions in tick physiology.

In seven other ixodid tick species we found $35 \alpha$-2Ms: I. scapularis (17), R. pulchellus (11), R. microplus (3), and one each for $I$. ricinus, A. maculatum, A. triste and A. parvum (Additional file 1: Table S1, Fig. 2). Our interspecific BLAST analyses showed four A. americanum $\alpha$ 2Ms are highly conserved in I. scapularis (Additional file 2: Table S2), with identities ranging from 76 to $83 \%$. These PIs appear to be widely distributed in males and females in many tissue and time points which could indicate they may not be involved in inhibiting tick host proteases. Notably however, three of these four were not found in MG tissues. Interestingly, we found one $A$. americanum $\alpha-2 \mathrm{M}$ with $68 \%$ identity to an $\alpha-2 \mathrm{M}$ in an argasid tick (Ornithodorous moubata, AAN10129). Such high identity values of metastriate $\alpha$-2Ms with prostriate and argasid tick $\alpha$ $2 \mathrm{Ms}$ indicates that at least some $\alpha-2 \mathrm{Ms}$ likely control fundamental pathways in tick physiology. Accordingly, these PIs warrant further consideration as potential anti-tick vaccine or druggable targets. Most $A$. americanum $\alpha$-2Ms however, did not show identity with any other tick $\alpha-2 \mathrm{M}$, suggesting significant divergence for some members of this family.

In both vertebrates and invertebrates, $\alpha-2 \mathrm{Ms}$ are considered components of the innate immune system involved in clearance of rogue endogenous and exogenous proteases [114, 115]. Emerging functional data show this is also the case in ticks. Indeed, one $\alpha 2 \mathrm{M}$ in $D$. variabilis (BQ426156) is differentially upregulated when this species is infected with Rickettsia montana [116]. In other studies, RNAi-mediated silencing of $I$. ricinus $\alpha 2 \mathrm{M}$ mRNA shows reduced phagocytosis of pathogens by tick cells, validating the significance of this protein family in the tick immune response [117]. The $\alpha 2 \mathrm{M}$-like protein from the ixodid tick I. ricinus has been characterized as functioning in pathogen phagocytosis by hemocytes in the hemolymph [117]. Similarly, the $\alpha 2 \mathrm{M}$ TAM (tick $\alpha$-macroglobulin) from Ornithodoros moubata is a trypsin inhibitor expressed by tick hemocytes and SG tissues [118, 119]. Collectively, this evidence suggests we may expect to find $\alpha 2 \mathrm{Ms}$ in all tick species. 


\section{Family 143: Opossum proteinase inhibitor (oprin)}

In $A$. americanum we found 34 non-redundant oprin sequences (Table 1). Of these 34, our transcriptome data confirm the expression of 11 in SG and 10 in MG tissues and nine found in both tissues, while almost half were not detected in these tissues. It will be interesting to use qRTPCR methods to validate the lack of expression of some oprin sequences in A. americanum SG and MG tissues. We also found 20 oprin sequences in unfed and 23 in fed ticks. These data imply that oprins serve diverse functional roles in tick physiology. Interestingly, we found seven sequences present only in SG and five only in MG, but that were not in males or in whole tick females. Based on these data it will be interesting to explore in future studies the hypothesis that these seven oprins are exclusively expressed in female SG or MG. As with CTLA sequences, we detected most oprin sequences in only one tissue at single feeding time point, which will be interesting to validated using qPCR analysis.

In other ixodid ticks we found 41 putatively nonredundant oprins and only in I. scapularis. While the MEROPS database indicates 22 oprins for $I$. scapularis (Additional file 1: Table S1), we found two sequences (MEROPS ID: MER218533 and MER218534) to be identical; therefore, MER218534 was eliminated from further analysis. Another MEROPS entry (MEROPS ID: MER160224) is concurrently listed in both the Kazal and oprin families. The domain profile of this protein contains a single Kazal domain and two Ig domain regions, and therefore may belong to two inhibitor families, or may be a mis-annotation. Inter-specific BLASTX searches of $A$. americanum oprin sequences revealed six homologs in $I$. scapularis with > 70\% identity and eight with 50-67\% identity (Additional file 2: Table S2). We found most of these highly conserved A. americanum oprins only in limited tissues and time points. For example, the top three conserved oprins at 83,82 and $80 \%$ identity with I. scapularis sequences were found only in MG at 48- and $120 \mathrm{~h}$, only in SG at $48 \mathrm{~h}$, and only in unfed males, respectively, however, these data should be further validated using qRT-PCR analysis.

Oprins are inhibitors of metalloendopeptidases and have varying numbers of immunoglobulin domains [120], but no defining domain profile. While several oprins function as snake venom metalloprotease inhibitors [120, 121], one oprin has been characterized as an immunoglobulin alpha FC receptor (FCalphaRI), which is the IgA receptor found on myeloid cells [122]. In this study, BLASTP scanning of tick sequences using the oprin type-entry from $D$. virginiana (MEROPS ID: MER019033), the alpha1B glycoprotein entry from humans (MEROPS ID: MER018491) and the Ig alpha FC receptor from humans (MEROPS ID: MER033169), revealed no tick sequences of significant similarity, indicating I43 proteins may serve different functional roles in ticks.

\section{Family 151: Phosphatidylethanolamine-binding proteins (PEBPs)}

In $A$. americanum we found 14 non-redundant phosphatidylethanolamine-binding protein (PEBP) sequences (Table 1). Our transcriptome data confirm expression of all 14 PEBPs in males, however nine were not found in females. The five PEBPs found in female ticks were also found in male ticks, which will be interesting to validate using qRT-PCR data since this would indicate females do not express any unique PEBPs. Since this is one of the smaller PI families, which could indicate low or no functional redundancy of PEBPs in ticks, it will be interesting to further investigate the function of the five PEPBs found in SG and MG tissues in this study.

In six other ixodid tick species we found 17 nonredundant PEBPs: seven for $I$. scapularis, four for $A$. maculatum, three for $I$. ricinus, and one each for $A$. triste, $A$. parvum and $A$. cajennense (Additional file 1: Table S1). Seven CDS have been reported in A. maculatum [45], and six can be found in GenBank, however only four of those six are non-redundant, therefore an exact count for A. maculatum remains to be determined. Data are less often collected for male ticks than for female ticks and this might explain why the number of known PEBPs in other tick species is less than in $A$. americanum. We found homologs to only three of $14 A$. americium PEBPs in other ticks and only in I. scapularis (Additional file 2: Table S2). However, the identities for these sequences ranged from 56 to $69 \%$. Interestingly, two of these three PEBPs were confirmed by our transcriptome data to be expressed in all tissues and feeding time points. Given the evolutionary distance between Amblyomma and Ixodes, we speculate homologs in metastriate tick species will be found when more data become available.

PEBPs are widespread among all types of organisms, however the function of PEBPs in various organisms is still being investigated. Some PEBPs have shown inhibitory function against thrombin, chymotrypsin and neuropsin [123]. Additionally, inhibition of the non-protease proteins Raf-1 kinase and G-protein-coupled receptor kinase 2 has been proposed [124]. The role of PEBPs in tick physiology has not yet been investigated beyond comparative transcriptomics. In D. variabilis, one putative PEBP was downregulated in the synganglion of replete-fed females, as compared to partially fed females [42]. More recently, PEBP transcripts were found in the salivary glands of three Amblyomma species: A. parvum, A. cajennense and A. triste [44]. These findings suggest PEBPs may play several different roles in tick physiology.

\section{Family 153: Madanins}

We found no madanin sequences in our A. americanum libraries. Family I53 members, known as madanins, were first discovered as blood coagulation inhibitors from $H$. 
longicornis [125], found in the salivary glands of feeding ticks [34]. This family has since been reported from the salivary proteome of $H$. marginatum rufipes, where a total of four sequences were annotated as madanin 1-4 [38], however none of these sequences show an I53 domain in a CDD search and these sequences show no identity to other madanin sequences and were therefore excluded them from our protease inhibitor counts. In $D$. andersoni four sequences have been identified as putative salivary madanin proteins [126] which we included in our counts, however sequences were not found in GenBank or UniProt for domain verification. In $H$. bispinosa two I53 domain sequences named haemathrin -1 and -2 have been deposited in GenBank (Additional file 1: Table S1).

Madanins are between 78 and 80 amino acids in length and share between 30 and $96 \%$ sequence identity (data not shown). Madanins have two unique features. One is that these sequences lack cysteine residues, which is relatively uncommon among thrombin inhibitors of blood feeding arthropods [127]. The other feature is two clusters of acidic residues in the $\mathrm{N}$-terminus of the sequence (first $2 / 3 \mathrm{~s}$ of the sequence) [127]. Crystallography studies show madanins bind with low affinity to thrombin and bind to thrombin's active site [127]. Madanins found in other tick species have not yet been characterized.

\section{Family 163: Pro-eosinophilic major basic protein (pro-MBP)}

In A. americanum we found 47 non-redundant pro-MBPs (Table 1). Our transcriptome data confirm expression of most (32/47) of these PIs in both male and female ticks, and most (30/47) in both fed and unfed ticks. Interestingly, we found eight pro-MBPs only in female ticks and eight only in male ticks. We also found nine pro-MBPs exclusively in each of unfed and fed ticks. Therefore, while it appears that most pro-MBPs in A. americanum are not sex- or time point-specific, it will be interesting to verify by qRT-PCR if there are some that are differentially expressed. While we found 48 total pro-MBP sequences in A. americanum, only 19 were found in SG and MG tissues, and it will be interesting to determine with qRT-PCR analysis if the other 27 are only expressed elsewhere in the tick.

We found one pro-MBP sequence for I. scapularis in MEROPS, but could not find pro-MBPs in any other tick species. Additional file 2: Table S2 outlines results from BLASTX scanning of $A$. americanum pro-MBPs against other tick sequences in GenBank. Eighteen of $47 \mathrm{~A}$. americanum sequences had homologs in I. scapularis, which led us to increase the pro-MBP count for I. scapularis to 14 putatively non-redundant sequences. What is striking is the very high similarity between I. scapularis and $A$. americanum pro-MBP sequences: four sequences had $63-66 \%$ identity, four sequences had $75-79 \%$ identity, five sequences had $80-87 \%$ identity and two sequences had
94-95\% identity to $I$. scapularis sequences. For ten of the 18 conserved $A$. americanum pro-MBPs, our transcriptome data could confirm expression in only a single tissue and time point, and six of these 10 were in unfed males only in our data. It will be interesting to further investigate the hypothesis of tissue and time point-specific expression of conserved $A$. americanum pro-MBPs.

In humans, pro-MBP has been shown to inhibit pregnancy-associated plasma protein-A (PAPP-A), which is a metalloprotease found both in a variety of normal tissues and in injured vasculature and skin, and is responsible for promoting cell growth and repair [128, 129]. Another I63 member in snake venom, bothrojaracin (Bothrops jararaca), has been characterized as a thrombin-induced platelet aggregation inhibitor, interacting with thrombin's exosite 1 to inhibit factor V activation [130, 131]. Interestingly, it has been noted that pro-MBP is capable of inhibiting MBP [132, 133], which is toxic to the schistosomulum of Schistosoma mansoni [132, 133], however, there have been no studies investigating the role of the pro-MBP protein in any tick species or other parasites.

\section{Family 168: Tick Carboxypeptidase Inhibitor (TCI)}

Family I68 sequences are known as tick carboxypeptidase inhibitors (tick CPI, or TCI). In A. americanum we found a single non-redundant TCI in GenBank (JAG91585) (Additional file 2: Table S2). In eight other ixodid tick species we found 23 putatively non-redundant TCI sequences: 10 for $R$. pulchellus, four for I. ricinus [134], three each for A. maculatum [45] and I. scapularis (MEROPS), and one each for $R$. bursa [135], R. sanguineus [39] and H. longicornis [136]; and (Additional file 1: Table S1). It is interesting to note the low family membership for TCIs, with most species having just one or a few non-redundant sequences. This could indicate that TCIs are functionally non-redundant and could be interesting targets for tick control. Rhipicephalus pulchellus is a notable exception, with 10 non-redundant TCIs. However, of the 10 non-redundant sequences our CDD searchers showed I68 domains in only eight. In $D$. andersoni six potential orthologs have been reported [137] but are not available in public databases for further analysis. The only TCI found for A. americanum showed $61 \%$ identity to the carboxypeptidase inhibitor in H. longicornis (ABO93460).

As the name suggests, TCIs are found exclusively in ticks and inhibit carboxypeptidases [50]. Functional studies show that the TCI in $R$. bursa inhibits proteases using its $\mathrm{C}$-terminus at the protease active site, and its $\mathrm{N}$-terminus at the carboxypeptidase exosite, and show a role for this protein in fibrinolysis acceleration by inhibiting thrombinactivatable fibrinolysis inhibitor (TAFI) [135]. TAFI also functions in wound healing, tissue remodeling and inflammation $[135,138]$. Additionally, TCIs from both $R$. bursa and $H$. longicornis have been demonstrated to inhibit 
carboxypeptidases A and B, which function in mast cellrelated inflammation, and in peptide digestion in pancreas and mast cells $[136,139,140]$. This is interesting because mast cells are suggested to be important in host defense against parasitism. Indeed, inhibitors of mast cell carboxypeptidase A from Ascaris suum have been concluded as a significant for the nematode's survival within its host $[140,141]$. More data are needed to further characterize the role of TCIs in tick physiology.

\subsection{Family 172: Chimadanins}

We found no chimadanins in A. americanum. Family I72 was established with the discovery of a blood coagulation inhibitor from $H$. longicornis [142]. This protein was named chimadanin and remains the sole member for this family, not having been discovered in any other organism (Additional file 1: Table S1). Chimadanin is a thrombin inhibitor of only 93 amino acids [142]. Expression of this protein is notable in the salivary glands of ticks during feeding [142]. This protein shows no domains in a CDD search. A recent study of the $R$. sanguineus sialotranscriptome resulted in a sequence bioinformatically annotated as chimadanin anti-thrombin like (ACX53883) [39], however a CDD search of this sequence showed an I53 domain. Thus, the placement of this sequence in family I72 is likely incorrect, leaving the chimadanin from $H$. longicornis as the sole I72 sequence. Due to the lack of a specific domain characterizing this family it may be difficult to assign new members to this family. Future assignment may be limited to sequences showing very high sequence conservation, followed by functional characterization studies.

\section{Family 174: Variegin}

Family I74, known as variegin, is comprised of a single sequence from one tick species, $A$. variegatum (Additional file 1: Table S1). As was the case in our $A$. americanum transcriptome analysis and in the $A$. triste, $A$. parvum and $A$. cajennense transcriptome analyses, studies may fail to find this protein because of its unusually small size of only 32 amino acids [44]. Like madanins, this sequence lacks cysteine residues [143]. Functional characterization of this protein shows that it is a thrombin inhibitor [144]. A prolonged inhibition of thrombin is interesting from the perspective of tick feeding which requires an interruption of blood coagulation for the many days over which tick feeding occurs. It will be interesting to see if further tick transcriptome analyses reveal this protein in other tick species.

\section{Conclusions}

Studies characterizing the function of tick PIs show a role in host protease regulation $[50,60,145,146]$, and in the regulation of tick proteases [55, 83]. This study shows that there are at least 1,595 known putative non-redundant PIs in ixodid tick species. Our $A$. americanum transcriptome analyses reveal that just six PI families: I2, I4, I25, I39, I43 and I63 represent the vast majority (80\%) of the A. americanum PI repertoire. Based on these results, we predict many more tick PIs are as yet undiscovered, primarily from one of the big three families: I2, I4 and I8. Though limited by the fact that transcripts in this study may not have been found due to technical and not biological reasons, our analysis of $A$. americium transcriptomes showed all PI families were always present, (with the exception of I35), but also supported previous in silico, semi-quantitiative and quantitative RT-PCR data showing differential expression of specific PIs within families [49, 51-59], between the sexes, tissues, and throughout feeding. It is not possible to study all 1,595 tick PIs in a reasonable time frame, therefore this study provides a prioritization template for selecting suitable anti-tick vaccine and/or pharmacologically relevant targets. The discovery of tick PIs that are conserved across different tick species [53] suggests some PIs likely regulate pathways that important to all ticks, and suggests these could be targeted for development of universal anti-tick vaccines. Conversely, PI families found neither in the I. scapularis genome, nor in our extensive transcriptome data for $A$. americanum, suggest we will find more PIs unique to certain tick species. These proteins are not attractive candidates for a broad-spectrum anti-tick vaccine. We propose that tick PI families with low PI numbers, suggesting non-redundancy in function, and that are highly conserved across species be among the priority proteins to investigate in future studies.

\section{Additional files}

Additional file 1: Table S1. Counts of protease inhibitors for ixodid tick species and GenBank accession numbers for accessed tick PI sequences by PI family. (XLSX $97 \mathrm{~kb}$ )

Additional file 2: Table S2. Results of Amblyomma americanum BLASTX search against tick sequences in the GenBank database. (XLSX 83 $\mathrm{kb})$

Additional file 3: FASTA sequences for Amblyomma americanum contigs from Illumina sequencing, by PI family. (ZIP 638 kb)

\section{Acknowledgements \\ We would like to thank Texas A\&M University for making freely available to our lab the license to the CLC software used in this study. \\ Funding \\ This research was supported by National Institutes of Health, USA grants (Al081093, Al093858, Al074789, Al074789-01A1S1) to AM.}

Availability of data and materials

Amblyomma americanum raw sequence read data are available in the NCBI Sequence Read Archive http://www.ncbi.nlm.nih.gov/sra and can be accessed with the following BioProject ID\# PRJNA226980. Contigs presented Additional file 3 have been deposited in DDBJ/EMBL/GenBank as a part of Transcriptome Shotgun Assembly project under the accession number GFBJ00000000. The version 
described in this paper is the first version, GFBJ01000000. Amblyomma americanum contigs analyzed in this study are provided in supplemental material by protease inhibitor family, excluding families 14 and 168 , which are available along with all sequences from other tick species in the GenBank repository http://www.ncbi.nlm. nih.gov/genbank/.

\section{Authors' contributions}

AM, LP and ZR designed the study. LP collected and analyzed data. LP and ZR designed figures and tables. LP and AM drafted the manuscript. All authors read and approved the final manuscript.

\section{Competing interests}

The authors declare that they have no competing interests.

\section{Consent for publication}

Not applicable.

\section{Ethics approval and consent to participate}

Not applicable.

\section{Publisher's Note}

Springer Nature remains neutral with regard to jurisdictional claims in published maps and institutional affiliations.

Received: 21 August 2016 Accepted: 6 March 2017

Published online: 22 March 2017

\section{References}

1. Sonenshine DE, Roe RM. Biology of ticks. 2nd ed. New York: Oxford University Press; 2014

2. Centers for Disease Control and Prevention: Tickborne Diseases of the United States. http://www.cdc.gov/ticks/diseases/. Accessed 1 Aug 2016.

3. Parola P, Raoult D. Ticks and tickborne bacterial diseases in humans: An emerging infectious threat. Clin Infect Dis. 2001;32:897-928.

4. Jongejan F, Uilenberg G. The global importance of ticks. Parasitology. 2004 129(Suppl):S3-14.

5. Reichard MV, Edwards AC, Meinkoth JH, Snider TA, Meinkoth KR, Heinz RE, et al. Confirmation of Amblyomma americanum (Acari: Ixodidae) as a vector for Cytauxzoon felis (Piroplasmorida: Theileriidae) to domestic cats. J Med Entomol. 2010:47:890-6.

6. Marcelino I, de Almeida AM, Ventosa M, Pruneau L, Meyer DF, Martinez D, et al. Tick-borne diseases in cattle: applications of proteomics to develop new generation vaccines. J Proteomics. 2012;75(14):4232-50.

7. Graf J, Gogolewski R, Leach-Bing N, Sabatini G, Molento M, Bordin E, et al. Tick control: An industry point of view. Parasitology. 2004;129(Suppl):427-42.

8. Willadsen P. Antigen cocktails: Valid hypothesis or unsubstantiated hope? Trends Parasitol. 2008:24:164-7.

9. de la Fuente J, Almazan C, Canales M, Perez de la Lastra JM, Kocan KM, Willadsen P. A ten-year review of commercial vaccine performance for control of tick infestations on cattle. Anim Health Res Rev. 2007;8:23-8.

10. George JE, Pound JM, Davey RB. Chemical control of ticks on cattle and the resistance of these parasites to acaricides. Parasitology. 2004;129(Suppl):353-66.

11. Shapiro SZ, Voigt WP, Fujisaki K. Tick antigens recognized by serum from a guinea pig resistant to infestation with the tick Rhipicephalus appendiculatus. J Parasitol. 1986;72:454-63.

12. Redondo M, Fragoso H, Montero C, Lona J, Medellín J, Fría R, et al. Integrated control of acaricide-resistant Boophilus microplus populations on grazing cattle in Mexico using vaccination with Gavac@ and amidine treatments. Exp Appl Acarol. 1999;23:841-9.

13. Demetz G, Ott I. The interface between inflammation and coagulation in cardiovascular disease. Int J Inflam. 2012;2012:860301.

14. Forneris F, Wu J, Gros P. The modular serine proteases of the complement cascade. Curr Opin Struct Biol. 2012;22:333-41.

15. Ichinose A. Factor XIII is a key molecule at the intersection of coagulation and fibrinolysis as well as inflammation and infection control. Int J Hematol. 2012;95:362-70

16. Loebbermann J, Thornton H, Durant L, Sparwasser T, Webster KE, Sprent J, et al. Regulatory $T$ cells expressing granzyme B play a critical role in controlling lung inflammation during acute viral infection. Mucosal Immunol. 2012;5:161-72.
17. Qureshi N, Morrison DC, Reis J. Proteasome protease mediated regulation of cytokine induction and inflammation. BBA-Mol Cell Res. 2012;1823(11):2087-93.

18. Zhou F, Zhang X, van Dam H, ten Dijke P, Huang H, Zhang L. Ubiquitinspecific protease 4 mitigates toll-like/interleukin-1 receptor signaling and regulates innate immune activation. J Biol Chem. 2012;287(14):11002-10.

19. Benson HL, Wilkes DS. Matrix metalloproteinases in T cell mediated pulmonary diseases. Front Biosci (Elite Ed). 2012;4:2162-9.

20. Bosche B, Molcanyi M, Noll T, Kochanek M, Kraus B, Rieger B, et al. Occurrence and recurrence of spontaneous chronic subdural haematoma is associated with a factor XIII deficiency. Clin Neurol Neurosurg. 2013;115(1):13-8.

21. López-Otín C, Bond JS. Proteases: Multifunctional enzymes in life and disease. J Biol Chem. 2008;283:30433-7.

22. Kanoh Y, Ohtani H, Egawa S, Baba S, Akahoshi T. Changes of proteases and proteinase inhibitors in androgen-dependent advanced prostate cancer patients with alpha2-macroglobulin deficiency. Clin Lab. 2012;58:217-25.

23. Mocchegiani E, Giacconi R, Costarelli L. Metalloproteases/anti-metalloproteases imbalance in chronic obstructive pulmonary disease: Genetic factors and treatment implications. Curr Opin Pulm Med. 2011;17 Suppl 1:11-9.

24. Mulenga A, Sugino M, Nakajima M, Sugimoto C, Onuma M. Tick-encoded serine proteinase inhibitors (serpins); potential target antigens for tick vaccine development. J Vet Med Sci. 2001;63:1063-9.

25. Mulenga A, Tsuda A, Sugimoto C, Onuma M. Blood meal acquisition by ticks; molecular advances and implications for vaccine development. Jpn J Vet Res. 2002;49:261-72.

26. Limo MK, Voigt WP, Tumbo-Oeri AG, Njogu RM, Ole-MoiYoi OK. Purification and characterization of an anticoagulant from the salivary glands of the ixodid tick Rhipicephalus appendiculatus. Exp Parasitol. 1991;72:418-29.

27. Vermeulen NM, Viljoen GJ, Bezuidenhout JD, Visser L, Neitz AW. Kinetic properties of toxic protease inhibitors isolated from tick eggs. Int J Biochem. 1988;20:621-31.

28. Willadsen P, McKenna RV. Trypsin-chymotrypsin inhibitors from the tick, Boophilus microplus. Aust J Exp Biol Med Sci. 1983;61(Pt 2):231-8.

29. Imamura S, da Silva Vaz Jr I, Sugino M, Ohashi K, Onuma M. A serine protease inhibitor (serpin) from Haemaphysalis longicornis as an anti-tick vaccine. Vaccine. 2005:23:1301-11.

30. Imamura S, Namangala B, Tajima T, Tembo ME, Yasuda J, Ohashi K, et al. Two serine protease inhibitors (serpins) that induce a bovine protective immune response against Rhipicephalus appendiculatus ticks. Vaccine. 2006;24:2230-7.

31. Lai R, Takeuchi H, Jonczy J, Rees HH, Turner PC. A thrombin inhibitor from the ixodid tick, Amblyomma hebraeum. Gene. Gene. 2004;342:243-9.

32. Mulenga $A$, Misao $O$, Sugimoto $C$. Three serine proteinases from midguts of the hard tick Rhipicephalus appendiculatus; cDNA cloning and preliminary characterization. Exp Appl Acarol. 2003;29:151-64.

33. Mulenga A, Tsuda A, Onuma M, Sugimoto C. Four serine proteinase inhibitors (serpin) from the brown ear tick, Rhiphicephalus appendiculatus; cDNA cloning and preliminary characterization. Insect Biochem Mol Biol. 2003;33:267-76.

34. Nakajima C, da Silva Vaz Jr I, Imamura S, Konnai S, Ohashi K, Onuma M. Random sequencing of cDNA library derived from partially-fed adult female Haemaphysalis longicornis salivary gland. J Vet Med Sci. 2005;67:1127-31.

35. Sugino M, Imamura S, Mulenga A, Nakajima M, Tsuda A, Ohashi K, et al. A serine proteinase inhibitor (serpin) from ixodid tick Haemaphysalis longicornis; cloning and preliminary assessment of its suitability as a candidate for a tick vaccine. Vaccine. 2003;21:2844-51.

36. Gulia-Nuss M, Nuss AB, Meyer JM, Sonenshine DE, Roe RM, Waterhouse RM, et al. Genomic insights into the Ixodes scapularis tick vector of Lyme disease. Nat Commun. 2016;7:10507.

37. Francischetti IM, Pham VM, Mans BJ, Andersen JF, Mather TN, Lane RS, et al. The transcriptome of the salivary glands of the female western black-legged tick Ixodes pacificus (Acari: Ixodidae). Insect Biochem Mol Biol. 2005;35:1142-61.

38. Francischetti IMB, Anderson JM, Manoukis N, Pham VM, Ribeiro JMC. An insight into the sialotranscriptome and proteome of the coarse bontlegged tick, Hyalomma marginatum rufipes. J Proteomics. 2011;74:2892-908.

39. Anatriello E, Ribeiro JM, de Miranda-Santos IK, Brandao LG, Anderson JM, Valenzuela JG, et al. An insight into the sialotranscriptome of the brown dog tick, Rhipicephalus sanguineus. BMC Genomics. 2010;11:450.

40. Anderson J, Valenzuela J. Tick saliva: from pharmacology and biochemistry to transcriptome analysis and functional genomics. In: Bowman AS, Nuttall P, editors. Ticks: biology, disease and control. New York: Cambridge University Press; 2008. p. 92-107.

41. Aljamali M, Hern L, Kupfer D, Downard S, So S, Roe B, et al. Transcriptome analysis of the salivary glands of the female tick Amblyomma americanum (Acari: Ixodidae). Insect Mol Biol. 2009;18:129-54. 
42. Bissinger B, Donohue K, Khalil S, Grozinger C, Sonenshine D, Zhu J, et al. Synganglion transcriptome and developmental global gene expression in adult females of the American dog tick, Dermacentor variabilis (Acari: Ixodidae). Insect Mol Biol. 2011;20:465-91.

43. Donohue KV, Khalil SM, Ross E, Grozinger CM, Sonenshine DE, Roe RM. Neuropeptide signaling sequences identified by pyrosequencing of the American dog tick synganglion transcriptome during blood feeding and reproduction. Insect Biochem Mol Biol. 2010;40:79-90.

44. Garcia GR, Gardinassi LG, Ribeiro JM, Anatriello E, Ferreira BR, Moreira HNS, et al. The sialotranscriptome of Amblyomma triste, Amblyomma parvum and Amblyomma cajennense ticks, uncovered by 454-based RNA-seq. Parasit Vectors. 2014;7:1.

45. Karim S, Singh P, Ribeiro JMC. A deep insight into the sialotranscriptome of the gulf coast tick, Amblyomma maculatum. PLoS One. 2011;6:e28525.

46. Pagel Van Zee J, Geraci NS, Guerrero FD, Wikel SK, Stuart JJ, Nene VM, et al. Tick genomics: The Ixodes genome project and beyond. Int J Parasitol. 2007; 37:1297-305.

47. Ribeiro JM, Anderson JM, Manoukis NC, Meng Z, Francischetti IM. A further insight into the sialome of the tropical bont tick, Amblyomma variegatum. BMC Genomics. 2011;12:136.

48. Richards SA, Stutzer C, Bosman A, Maritz-Olivier C. Transmembrane proteins - mining the cattle tick transcriptome. Ticks Tick Borne Dis. 2015;6:695-710.

49. Tan AW, Francischetti IM, Slovak M, Kini RM, Ribeiro JM. Sexual differences in the sialomes of the zebra tick, Rhipicephalus pulchellus. J Proteomics. 2015;117:120-44.

50. Rawlings ND, Barrett AJ, Bateman A. MEROPS: The database of proteolytic enzymes, their substrates and inhibitors. Nucleic Acids Res. 2012;40:D343-50.

51. De Castro MH, De Klerk D, Pienaar R, Latif AA, Rees DJ, Mans BJ. De novo assembly and annotation of the salivary gland transcriptome of Rhipicephalus appendiculatus male and female ticks during blood feeding. Ticks Tick Borne Dis. 2016;7(4):536-48.

52. Karim S, Ribeiro JMC. An insight into the sialome of the Lone Star tick, Amblyomma americanum, with a glimpse on its time dependent gene expression. PLoS One. 2015;10:e0131292.

53. Porter L, Radulović Ž, Kim T, Braz GR, da Silva Vaz Jr I, Mulenga A. Bioinformatic analyses of male and female Amblyomma americanum tick expressed serine protease inhibitors (serpins). Ticks Tick Borne Dis. 2015;6:16-30.

54. Cao J, Shi L, Zhou Y, Gao X, Zhang H, Gong H, et al. Characterization of a new Kunitz-type serine protease inhibitor from the hard tick Rhipicephalus hemaphysaloides. Arch Insect Biochem Physiol. 2013;84:104-13.

55. Lu S, Soares TS, da Silva Vaz Junior I, Lovato DV, Tanaka AS. Rmcystatin3, a cysteine protease inhibitor from Rhipicephalus microplus hemocytes involved in immune response. Biochimie. 2014;106:17-23.

56. Liu XY, de la Fuente J, Cote M, Galindo RC, Moutailler S, Vayssier-Taussat M, et al. IrSPI, a tick serine protease inhibitor involved in tick feeding and Bartonella henselae infection. PLoS Negl Trop Dis. 2014;8(7):e2993.

57. Tirloni L, Seixas A, Mulenga A, da Silva VI, Termignoni C. A family of serine protease inhibitors (serpins) in the cattle tick Rhipicephalus (Boophilus) microplus. Exp Parasitol. 2014;137:25-34.

58. Rodriguez-Valle M, Xu T, Kurscheid S, Lew-Tabor AE. Rhipicephalus microplus serine protease inhibitor family: annotation, expression and functional characterisation assessment. Parasit Vectors. 2015;8(1):7.

59. Mulenga A, Khumthong R, Blandon MA. Molecular and expression analysis of a family of the Amblyomma americanum tick Lospins. J Exp Biol. 2007; 210(18):3188-98

60. Mulenga A, Kim T, Ibelli AMG. Amblyomma americanum tick saliva serine protease inhibitor 6 is a cross-class inhibitor of serine proteases and papain-like cysteine proteases that delays plasma clotting and inhibits platelet aggregation. Insect Mol Biol. 2013;22:306-19.

61. Zhou J, Liao M, Hatta T, Tanaka M, Xuan X, Fujisaki K. Identification of a follistatin-related protein from the tick Haemaphysalis longicornis and its effect on tick oviposition. Gene. 2006;372:191-8.

62. Munz B, Hubner G, Tretter Y, Alzheimer C, Werner S. A novel role of activin in inflammation and repair. J Endocrinol. 1999;161:187-93.

63. Phillips DJ, de Kretser DM, Hedger MP. Activin and related proteins in inflammation: Not just interested bystanders. Cytokine Growth Factor Rev. 2009;20:153-64.

64. Baskova IP, Zavalova LL. Proteinase inhibitors from the medicinal leech Hirudo medicinalis. Biochem Mosc. 2001;66:703-14.

65. Mende K, Lange U, Nowak G. Three recombinant serine proteinase inhibitors expressed from the coding region of the thrombin inhibitor dipetalogastin. Insect Biochem Mol Biol. 2004;34:971-9.
66. Campos ITN, Amino R, Sampaio CAM, Auerswald EA, Friedrich T, Lemaire $H G$, et al. Infestin, a thrombin inhibitor presents in Triatoma infestans midgut, a Chagas' disease vector: Gene cloning, expression and characterization of the inhibitor. Insect Biochem Mol Biol. 2002;32:991-7.

67. Rimphanitchayakit V, Tassanakajon A. Structure and function of invertebrate kazal-type serine proteinase inhibitors. Dev Comp Immunol. 2010;34:377-86.

68. Mulenga A, Khumthong R, Chalaire KC, Strey O, Teel P. Molecular and biological characterization of the Amblyomma americanum organic anion transporter polypeptide. J Exp Biol. 2008;211:3401-8.

69. Radulović Ž, Porter LM, Kim TK, Mulenga A. Comparative bioinformatics, temporal and spatial expression analyses of Ixodes scapularis organic anion transporting polypeptides. Ticks Tick Borne Dis. 2014;5:287-98.

70. Koh CY, Kini RM. Molecular diversity of anticoagulants from haematophagous animals. Thromb Haemost. 2009;102:437-53.

71. Mans BJ, Louw Al, Neitz AWH. Evolution of hematophagy in ticks: Common origins for blood coagulation and platelet aggregation inhibitors from soft ticks of the genus Ornithodoros. Mol Biol Evol. 2002;19:1695-705.

72. Milstone AM, Harrison LM, Bungiro RD, Kuzmic P, Cappello M. A broad spectrum Kunitz type serine protease inhibitor secreted by the hookworm Ancylostoma ceylanicum. J Biol Chem. 2000;275:29391-9.

73. Ribeiro JMC, Mans BJ, Arcà B. An insight into the sialome of blood-feeding Nematocera. Insect Biochem Mol Biol. 2010;40:767-84.

74. Francischetti IMB, Valenzuela JG, Andersen JF, Mather TN, Ribeiro JMC. Ixolaris, a novel recombinant tissue factor pathway inhibitor (TFPI) from the salivary gland of the tick, Ixodes scapularis: Identification of factor $\mathrm{X}$ and factor $X a$ as scaffolds for the inhibition of factor Vlla/tissue factor complex. Blood. 2002;99:3602-12.

75. Francischetti IM, Mather TN, Ribeiro JM. Penthalaris, a novel recombinant fiveKunitz tissue factor pathway inhibitor (TFPI) from the salivary gland of the tick vector of Lyme disease, Ixodes scapularis. Thromb Haemost. 2004;91:886-98.

76. Mans BJ, Louw Al, Neitz AW. Savignygrin, a platelet aggregation inhibitor from the soft tick Ornithodoros savignyi, presents the RGD integrin recognition motif on the Kunitz-BPTI fold. J Biol Chem. 2002;277:21371-8.

77. Karczewski J, Endris R, Connolly TM. Disagregin is a fibrinogen receptor antagonist lacking the arg-gly-asp sequence from the tick, Ornithodoros moubata. J Biol Chem. 1994;269:6702-8.

78. Karczewski J, Connolly TM. The interaction of disagregin with the platelet fibrinogen receptor, glycoprotein IIb-llla. Biochem Biophys Res Commun. 1997;241:744-8.

79. Nienaber J, Gaspar ARM, Neitz AWH. Savignin, a potent thrombin inhibitor isolated from the salivary glands of the tick Ornithodoros savignyi (Acari: Argasidae). Exp Parasitol. 1999;93:82-91.

80. Macedo-Ribeiro S, Almeida C, Calisto B, Friedrich T, Mentele R, Stürzebecher $J$, et al. Isolation, cloning and structural characterisation of boophilin, a multifunctional Kunitz-type proteinase inhibitor from the cattle tick. PLoS One. 2008:3:e1624.

81. Lai R, Takeuchi H, Jonczy J, Rees HH, Turner PC. A thrombin inhibitor from the ixodid tick, Amblyomma hebraeum. Gene. 2004;342:243-9.

82. Liao M, Zhou J, Gong H, Boldbaatar D, Shirafuji R, Battur B, et al. Hemalin, a thrombin inhibitor isolated from a midgut cDNA library from the hard tick Haemaphysalis longicornis. J Insect Physiol. 2009;55:165-74.

83. Ceraul SM, Dreher-Lesnick SM, Mulenga A, Rahman MS, Azad AF. Functional characterization and novel rickettsiostatic effects of a Kunitz-type serine protease inhibitor from the tick Dermacentor variabilis. Infect Immun. 2008;76:5429-35.

84. Hunt LT, Dayhoff MO. A surprising new protein superfamily containing ovalbumin, antithrombin-III, and alpha1-proteinase inhibitor. Biochem Biophys Res Commun. 1980;95:864-71.

85. Hwang SR, Steineckert B, Toneff T, Bundey R, Logvinova AV, Goldsmith P, et al. The novel serpin endopin 2 demonstrates cross-class inhibition of papain and elastase: Localization of endopin 2 to regulated secretory vesicles of neuroendocrine chromaffin cells. Biochemistry. 2002:41:10397-405.

86. Hwang $\mathrm{S}$, Hook VYH. Multiple domains of endopin $2 \mathrm{~A}$ for serpin cross-class inhibition of papain. Arch Biochem Biophys. 2007:461:219-24.

87. Masumoto K, Sakata Y, Arima K, Nakao I, Izuhara K. Inhibitory mechanism of a cross-class serpin, the squamous cell carcinoma antigen 1. J Biol Chem. 2003;278:45296-304

88. Fogaça AC, Almeida IC, Eberlin MN, Tanaka AS, Bulet P, Daffre S. Ixodidin, a novel antimicrobial peptide from the hemocytes of the cattle tick Boophilus microplus with inhibitory activity against serine proteinases. Peptides. 2006; 27:667-74. 
89. Sasaki SD, de Lima CA, Lovato DV, Juliano MA, Torquato RJS, Tanaka AS. BmSl-7, a novel subtilisin inhibitor from Boophilus microplus, with activity toward $\operatorname{Pr} 1$ proteases from the fungus Metarhizium anisopliae. Exp Parasitol. 2008;118:214-20.

90. McNally KL, Mitzel DN, Anderson JM, Ribeiro JMC, Valenzuela JG, Myers TG, et al. Differential salivary gland transcript expression profile in Ixodes scapularis nymphs upon feeding or flavivirus infection. Ticks Tick Borne Dis. 2012;3:18-26.

91. Rudenko N, Golovchenko M, Edwards MJ, Grubhoffer L. Differential expression of Ixodes ricinus tick genes induced by blood feeding or Borrelia burgdorferi infection. J Med Entomol. 2005;42:36-41.

92. Nishio K, Anderson PJ, Zheng XL, Sadler JE. Binding of platelet glycoprotein iba to von willebrand factor domain A1 stimulates the cleavage of the adjacent domain A2 by ADAMTS13. Proc Natl Acad Sci USA. 2004;101: 10578-83.

93. Martens GJ, Braks JA, Eib DW, Zhou Y, Lindberg I. The neuroendocrine polypeptide 7B2 is an endogenous inhibitor of prohormone convertase PC2. Proc Natl Acad Sci USA. 1994;91:5784-7.

94. Karim S, Miller NJ, Valenzuela J, Sauer JR, Mather TN. RNAi-mediated gene silencing to assess the role of synaptobrevin and cystatin in tick blood feeding. Biochem Biophys Res Commun. 2005;334:1336-42.

95. Zhou J, Ueda M, Umemiya R, Battsetseg B, Boldbaatar D, Xuan X, et al. A secreted cystatin from the tick Haemaphysalis longicornis and its distinct expression patterns in relation to innate immunity. Insect Biochem Mol Biol. 2006:36:527-35.

96. Kotsyfakis M, Sá-Nunes A, Francischetti IM, Mather TN, Andersen JF, Ribeiro JM. Antiinflammatory and immunosuppressive activity of sialostatin L, a salivary cystatin from the tick Ixodes scapularis. J Biol Chem. 2006;281:26298-307.

97. Deshapriya RMC, Takeuchi A, Shirao K, Isa K, Watabe S, Murakami R, et al. Drosophila CTLA-2-like protein (D/CTLA-2) inhibits cysteine proteinase 1 (CP1), a cathepsin L-like enzyme. Zool Sci. 2007;24:21-30.

98. Kurata M, Hirata M, Watabe S, Miyake M, Takahashi SY, Yamamoto Y. Expression, purification, and inhibitory activities of mouse cytotoxic Tlymphocyte antigen-2a. Protein Expr Purif. 2003;32:119-25.

99. Yamamoto Y, Watabe S, Kageyama T, Takahashi SY. A novel inhibito protein for Bombyx cysteine proteinase is homologous to propeptide regions of cysteine proteinases. FEBS Lett. 1999:448:257-60.

100. Fujimoto Y, Kobayashi A, Kurata S, Natori S. Two subunits of the insect 26/ $29-\mathrm{kDa}$ proteinase are probably derived from a common precursor protein. J Biochem. 1999;125:566-73.

101. Lenarčič B, Ritonja A, Štrukelj B, Turk B, Turk V. Equistatin, a new inhibitor of cysteine proteinases from Actinia equina, is structurally related to thyroglobulin type-1 domain. J Biol Chem. 1997;272:13899-903.

102. Lenarčič B, Turk V. Thyroglobulin type-1 domains in equistatin inhibit both papain-like cysteine proteinases and cathepsin D. J Biol Chem. 1999;274: 563-6.

103. Morabito MA, Moczydlowski E. Molecular cloning of bullfrog saxiphilin: A unique relative of the transferrin family that binds saxitoxin. Proc Natl Acad Sci USA. 1994;91:2478-82.

104. Bocock JP, Edgell CS, Marr HS, Erickson AH. Human proteoglycan testican-1 inhibits the lysosomal cysteine protease cathepsin L. Eur J Biochem. 2003; 270:4008-15.

105. Aumailley M, Battaglia C, Mayer U, Reinhardt D, Nischt R, Timpl R, et al. Nidogen mediates the formation of ternary complexes of basement membrane components. Kidney Int. 1993;43:7-12.

106. Kim S, Wadsworth WG. Positioning of longitudinal nerves in C. elegans by nidogen. Science. 2000;288:150-4.

107. Deveraux QL, Reed JC. IAP family proteins - suppressors of apoptosis. Genes Dev. 1999;13:239-52

108. Deveraux QL, Stennicke HR, Salvesen GS, Reed JC. Endogenous inhibitors of caspases. J Clin Immunol. 1999;19:388-98.

109. Lewis SM, Holcik M. IRES in distress: Translational regulation of the inhibitor of apoptosis proteins XIAP and HIAP2 during cell stress. Cell Death Differ. 2005; 12:547-53.

110. Cawston TE, Galloway WA, Mercer E, Murphy G, Reynolds JJ. Purification of rabbit bone inhibitor of collagenase. Biochem J. 1981;195:159-65.

111. Visse R, Nagase H. Matrix metalloproteinases and tissue inhibitors of metalloproteinases. Circ Res. 2003;92:827-39.

112. Yong W, Power C, Forsyth $P$, Edwards DR. Metalloproteinases in biology and pathology of the nervous system. Nat Rev Neurosci. 2001;2:502-11.
113. Kuvaja P, Hulkkonen S, Pasanen I, Soini Y, Lehtonen S, Talvensaari-Mattila A, et al. Tumor tissue inhibitor of metalloproteinases-1 (TIMP-1) in hormoneindependent breast cancer might originate in stromal cells, and improves stratification of prognosis together with nodal status. Exp Cell Res. 2012;318: 1094-103.

114. Armstrong PB, Quigley JP. a2-macroglobulin: An evolutionarily conserved arm of the innate immune system. Dev Comp Immunol. 1999;23:375-90.

115. Scharfstein J. Parasite cysteine proteinase interactions with a2-macroglobulin or kininogens: Differential pathways modulating inflammation and innate immunity in infection by pathogenic trypanosomatids. Immunobiology. 2006; 211:117-25.

116. Mulenga A, Macaluso KR, Simser JA, Azad AF. Dynamics of Rickettsia-tick interactions: identification and characterization of differentially expressed mRNAs in uninfected and infected Dermacentor variabilis. Insect Mol Biol. 2003:12(2):185-93.

117. Burešová V, Hajdušek O, Franta Z, Sojka D, Kopáček P. IrAM—An a2-macroglobulin from the hard tick Ixodes ricinus: Characterization and function in phagocytosis of a potential pathogen Chryseobacterium indologenes. Dev Comp Immunol. 2009;33: 489-98.

118. Saravanan T, Weise C, Sojka D, Kopáček P. Molecular cloning, structure and bait region splice variants of a2-macroglobulin from the soft tick Ornithodoros moubata. Insect Biochem Mol Biol. 2003;33:841-51.

119. Kopáček P, Weise C, Saravanan T, Vítová K, Grubhoffer L. Characterization of an a-macroglobulin-like glycoprotein isolated from the plasma of the soft tick Ornithodoros moubata. Eur J Biochem. 2000;267:465-75.

120. Catanese JJ, Kress LF. Isolation from opossum serum of a metalloproteinase inhibitor homologous to human alpha 1B-glycoprotein. Biochemistry. 1992; 31:410-8.

121. Thwin M, Gopalakrishnakone P. Snake envenomation and protective natural endogenous proteins: A mini review of the recent developments (1991-1997). Toxicon. 1998;36:1471-82

122. Lang ML, Chen YW, Shen L, Gao H, Lang GA, Wade TK, et al. IgA fc receptor (FcalphaR) cross-linking recruits tyrosine kinases, phosphoinositide kinases and serine/threonine kinases to glycolipid rafts. Biochem J. 2002;364:517-25.

123. Hengst U, Albrecht H, Hess D, Monard D. The phosphatidylethanolaminebinding protein is the prototype of a novel family of serine protease inhibitors. J Biolo Chem. 2001;276:535-40.

124. Mima J, Fukada H, Nagayama M, Ueda M. Specific membrane binding of the carboxypeptidase $Y$ inhibitor IC, a phosphatidylethanolamine-binding protein family member. FEBS J. 2006;273:5374-83.

125. Iwanaga S, Okada M, Isawa H, Morita A, Yuda M, Chinzei Y. Identification and characterization of novel salivary thrombin inhibitors from the Ixodidae tick, Haemaphysalis longicornis. Eur J Biochem. 2003;270: 1926-34.

126. Francischetti IM, Sá-Nunes A, Mans BJ, Santos IM, Ribeiro JM. The role of saliva in tick feeding. Front Biosci (Landmark Ed). 2009;14:2051-88.

127. Figueiredo AC, de Sanctis D, Pereira PJB. The tick-derived anticoagulant madanin is processed by thrombin and factor Xa. PLoS One. 2013;8(8):e71866.

128. Boldt HB, Conover CA. Pregnancy-associated plasma protein-A (PAPP-A): A local regulator of IGF bioavailability through cleavage of IGFBPs. Growth Horm IGF Res. 2007:17:10-8.

129. Oxvig C, Sand O, Kristensen T, Gleich GJ, Sottrup-Jensen L. Circulating human pregnancy-associated plasma protein-A is disulfide-bridged to the proform of eosinophil major basic protein. J Biol Chem. 1993;268:12243-6.

130. Arocas V, Lemaire C, Bouton MC, Bezeaud A, Bon C, Guillin MC, et al. Inhibition of thrombin-catalyzed factor $V$ activation by bothrojaracin. Thromb Haemost. 1998;79:1157-61.

131. Zingali RB, Jandrot-Perrus M, Guillin MC, Bon C. Bothrojaracin, a new thrombin inhibitor isolated from Bothrops jararaca venom: Characterization and mechanism of thrombin inhibition. Biochemistry. 1993;32:10794-802.

132. McGrogan M, Simonsen C, Scott R, Griffith J, Ellis N, Kennedy J, et al. Isolation of a complementary DNA clone encoding a precursor to human eosinophil major basic protein. J Exp Med. 1988;168:2295-308.

133. Popken-Harris P, Thomas L, Oxvigc C, Sottrup-Jensenc L, Kuboa H, Kleind JS, et al. Biochemical properties, activities, and presence in biologic fluids of eosinophil granule major basic protein. J Allergy Clin Immunol. 1994;94:1282-9.

134. Chmelař J, Anderson J, Mu J, Jochim R, Valenzuela J, Kopecký J. Insight into the sialome of the castor bean tick, Ixodes ricinus. BMC Genomics. 2008:9:1-21.

135. Arolas JL, Lorenzo J, Rovira A, Castellà J, Aviles FX, Sommerhoff CP. A carboxypeptidase inhibitor from the tick Rhipicephalus bursa. J Biol Chem. 2005;280:3441-8. 
136. Gong H, Zhou J, Liao M, Hatta T, Harnnoi T, Umemiya R, et al. Characterization of a carboxypeptidase inhibitor from the tick Haemaphysalis longicornis. J Insect Physiol. 2007;53:1079-87.

137. Alarcon-Chaidez FJ, Sun J, Wikel SK. Transcriptome analysis of the salivary glands of Dermacentor andersoni stiles (Acari: Ixodidae). Insect Biochem Mol Biol. 2007;37:48-71.

138. Sanglas L, Valnickova Z, Arolas JL, Pallarés I, Guevara T, Solà M, et al. Structure of activated thrombin-activatable fibrinolysis inhibitor, a molecular link between coagulation and fibrinolysis. Mol Cell. 2008;31:598-606.

139. Reznik SE, Fricker LD. Carboxypeptidases from A to Z: Implications in embryonic development and wnt binding. Cell Mol Life Sci. 2001;58:1790-804.

140. Sanglas L, Aviles FX, Huber R, Gomis-Rüth FX, Arolas JL. Mammalian metallopeptidase inhibition at the defense barrier of Ascaris parasite. Proc Natl Acad Sci USA. 2009;106:1743-7.

141. Pejler G, Knight SD, Henningsson F, Wernersson S. Novel insights into the biological function of mast cell carboxypeptidase A. Trends Immunol. 2009; 30:401-8.

142. Nakajima C, Imamura S, Konnai S, Yamada S, Nishikado H, Ohashi K, et al. A novel gene encoding a thrombin inhibitory protein in a CDNA library from Haemaphysalis longicornis salivary gland. J Vet Med Sci. 2006;68:447-52.

143. Koh CY, Kazimirova M, Trimnell A, Takac P, Labuda M, Nuttall PA, et al. Variegin, a novel fast and tight binding thrombin inhibitor from the tropical bont tick. J Biol Chem. 2007;282:29101-13.

144. Koh CY, Kazimirova M, Nuttall PA, Kini RM. Noncompetitive inhibitor of thrombin. ChemBioChem. 2009;10:2155-8.

145. Chalaire KC, Kim TK, Garcia-Rodriguez H, Mulenga A. Amblyomma americanum (L.) (acari: Ixodidae) tick salivary gland serine protease inhibitor (serpin) 6 is secreted into tick saliva during tick feeding. J Exp Biol. 2011;214:665-73.

146. Valdés JJ, Schwarz A, de Vaca IC, Calvo E, Pedra JH, Guallar V, et al. Tryptogalinin is a tick Kunitz serine protease inhibitor with a unique intrinsic disorder. PLoS One. 2013;8:e62562.

\section{Submit your next manuscript to BioMed Central and we will help you at every step:}

- We accept pre-submission inquiries

- Our selector tool helps you to find the most relevant journal

- We provide round the clock customer support

- Convenient online submission

- Thorough peer review

- Inclusion in PubMed and all major indexing services

- Maximum visibility for your research

Submit your manuscript at www.biomedcentral.com/submit 